\title{
Pediatric Nodular Fasciitis in the Head and Neck Region
}

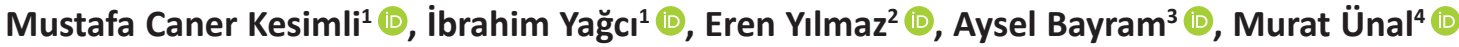

\author{
${ }^{1}$ Istinye University, Faculty of Medicine, Department of Otolaryngology, Head and Neck Surgery, Istanbul, Turkey \\ ${ }^{2}$ Gelişim University, Faculty of Medicine, Department of Otolaryngology, Head and Neck Surgery, Istanbul, Turkey \\ ${ }^{4}$ Istanbul University, Faculty of Medicine, Department of Pathology, Istanbul, Turkey \\ ${ }^{5}$ Mersin University, Facultyof Medicine, Department of Otolaryngology, Head and Neck Surgery, Mersin, Turkey
}

ORCID ID: M.C.K. 0000-0003-1675-0394; i.Y. 0000-0003-2039-8362; E.Y. 0000-0002-5349-9699; A.B. 0000-0002-5014-0074; M.Ü. 0000-0002-5524-9175

Citation: Kesimli MC, Yagci I, Yilmaz E, Bayram A, Unal M. Pediatric nodular fasciitis in the head and neck region. Tr-ENT 2021;31(4):105-107. https://doi.org/10.26650/Tr-ENT.2021.1026815

\section{ABSTRACT}

Nodular fasciit is a rare benign tumor and may occur anywhere in the body. Pediatric patients constitute approximately $10 \%$ of the entire patient group.

The disease may show clinical and pathological features resembling malignancy. Therefore, it can be mistaken as sarcoma.

This report shows two cases of pediatric nodular fasciitis localized in the parotid region and maxillary sinus.

Keywords: Nodular fasciitis, Spindle cell tumors, Maxillofacial region, Head and neck

\section{INTRODUCTION}

Nodular fasciitis (NF) is an extremely rare benign tumor that is localized in the subcutaneous tissue and presents as a mass (1). It was first defined by Konwaller in 1955 as "subcutaneous pseudosarcomatous fibromatosis" (1). NF usually presents as painless, rapidly growing solitary tumors and is most common in the third and fourth decades (1). While tumor localization in NF is most common in the upper extremity (48\%), it is less frequent in the trunk (20\%), lower extremities (15\%) and head and neck $(15-20 \%)(1,2)$.

The disease may show clinical and pathological features resembling malignancy, such as increased mitotic activity and rapid growth. For this reason, it can be confused with sarcoma (2). Therefore, a definitive diagnosis of the lesion should be made to avoid unnecessarily aggressive or inappropriate treatment. To increase awareness of NF as a differential diagnosis in rapid-growing solitary tumours in the head and neck region, we present two cases of NF the first, occurring in the parotid region, and the second in the maxillary sinus.

\section{CASE REPORTS}

\section{Case-1}

A 5-year-old male patient applied to our clinic complaining of facial swelling in the right parotid region which had progressed rapidly during a period of 3 months to the size of $3 \times 2 \mathrm{~cm}$ (Figure 1). The

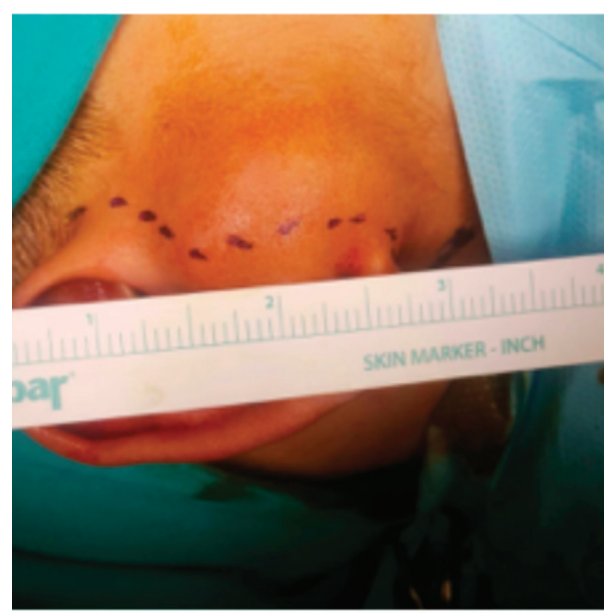

Figure 1: Right facial swelling in the pre-auricular region.

Corresponding Author: Mustafa Caner Kesimli E-mail: canerkesimli@gmail.com

Submitted: 11.01.2022 • Accepted: 18.01.2022 • Published Online: 28.01.2022 
patient's medical history was unremarkable. The patient had no history of trauma or surgery.

The tumor was a mobile, firm mass palpable in the subcutaneous tissue of the left cheek. There was no facial nerve paralysis or paraesthesia and no local or regional enlarged lymph nodes.

Fine needle aspiration (FNA) cytological analysis revealed a neuronal tumor. Magnetic resonance imaging showed the presence of a round, intraparotid, solid nodule; no other masses or lymph nodes were detected.

A superficial parotidectomy was planned. Following a right modified Blair incision, the skin and superficial musculoaponeurotic system were elevated over the tumor. The mass was located at the middle border of the parotid gland sheath, so the surgical plan was changed to tumor excision. The mass was dissected from the parotid tissue and then removed. The skin was sutured. The post-operative hospital stay was uneventful.

The histopathologic report showed a moderately cellular tumor with a hypocellular spindle cell component with collagenous stroma (Figure 2). Immunohistochemical stains were negative for beta-catenin (fibromatosis marker), desmin (smooth muscle marker), S-100 protein (neural marker) with a slight increase in the proliferation marker KI-67. Such findings were thought to be indicative of nodular fasciitis. Over a 24-month followup period, ultrasonography (USG) findings were periodically evaluated and no recurrence was detected.

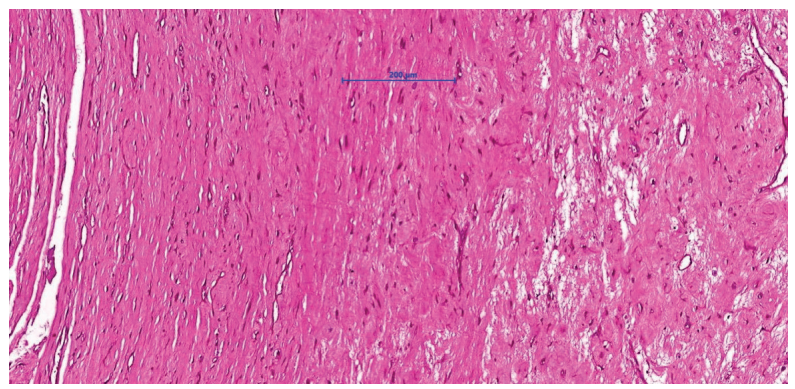

Figure 2: Hypocellular spindle cell tumour component with collagenous stroma (H-E, x100).

The patient's parents provided written consent after being informed about the procedures and aim of the study.

\section{Case 2}

A 6-year-old boy was admitted to our department after his parents noticed a facial fullness in the right maxillary region. They had also noticed a slight swelling at the right nasal sidewall, which had progressed rapidly during a period of 2 months. The medical and surgical history was normal and the patient had no history of trauma or surgery.

Examination revealed a $5 \times 4 \mathrm{~cm}$ solid mass at the right maxillary area extending to the right nasal cavity. Nasal mucosa was normal; however, the inferior turbinate was pushed medially and compressed on the right side. A CT scan and MRI showed a right maxillary well-circumscribed mass, measuring $5 \times 4.1 \mathrm{~cm}$.

A mid-facial degloving procedure was performed. After right sub-labial incision, the mass was removed from the maxillar sinus cavity (Figure 3). Incisions were closed with appropriate sutures. The post-operative stay was normal.

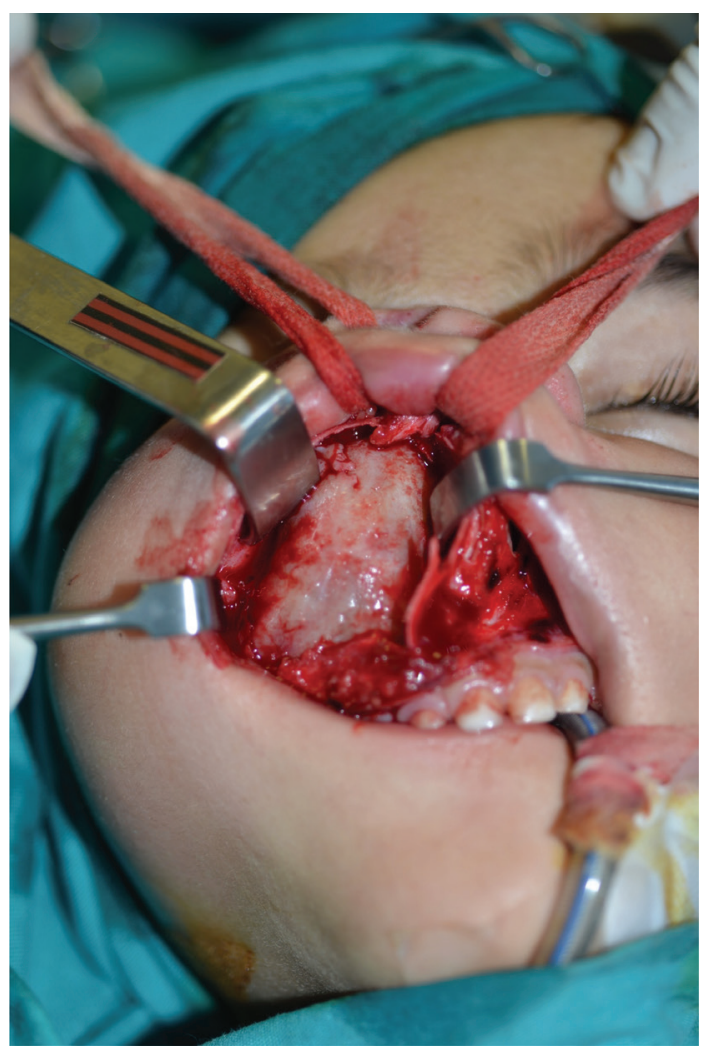

Figure 3: Right side well-circumscribried maxillary mass.

The histopathologic report showed a moderately cellular lesion comprising of proliferating spindle cells with collagenized stroma and focal myxomatous areas (Figure 4).

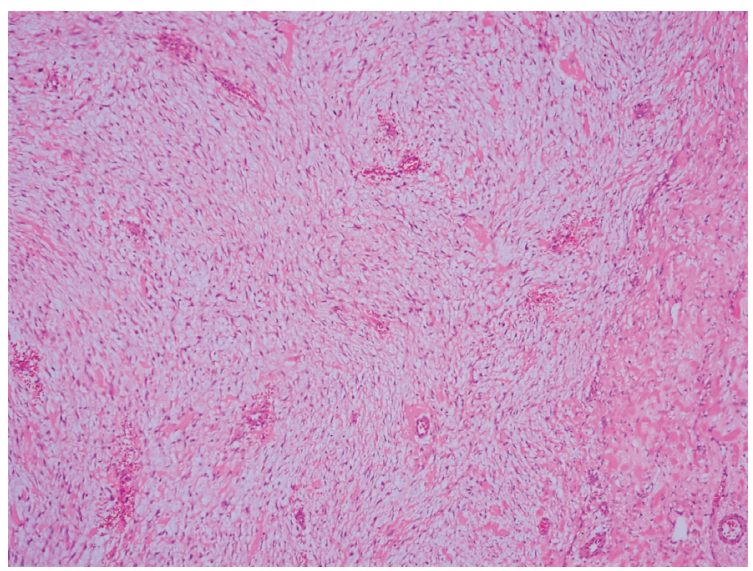

Figure 4: Proliferation of fibroblastic/myofibroblastic spindle cells within myxoid and collagenous stroma (Hematoxylin and eosin, x100). 
Immunohistochemical stains were negative for sarcoma and diagnosis was accepted as NF.

Over an 18-month follow-up period, magnetic resonance imaging (MRI) findings were regularly evaluated and no recurrence was detected.

His parents provided written consent after being informed about the procedures and aim of the study.

\section{DISCUSSION}

The prevalence of NF in children is rare with $10 \%$ of cases being observed in pediatric patients (3). In pediatric patients, unlike in adult patients, tumor location is most commonly reported in the head and neck region: in the facial skin, scalp, tongue, mandible, cheek mucosa, throat and floor of the mouth $(2,3)$. Paranasal sinus involvement is extremely rare (2). In our series one patient had a tumor in the parotid region and the other was in the maxillary sinus.

The etiology of this hyperproliferative tumor is unknown. Formerly, trauma was believed to play an important role. However, in recent years Erickson-Johnson et al. found NF is associated with MYHP-USP6 gene fusion (4). In our cases, there was no history of trauma.

NF usually presents as a rapid growing mass and mimics malignancy when it occurs in the head and neck region. Nonspecific imaging findings often contribute to this misdiagnosis. Differential diagnosis is important to rule out other malignancies, especially sarcomas, schwannomas and salivary gland tumours (1-3). Fibrosarcoma can be differentiated from NF by its less myxoid matrix mitoses and higher hypercellularity (2). Schwannomas are well-defined, round and encapsulated. Histologic features typically include palisades and Antoni A/B areas. Immunohistochemical stains show S100 positivity and can confirm the epidermal origin of schwannoma (2). In Pleomorphic adenoma a spindle cell presence is similarly seen in a myxoid stroma with strong actin positivity, however, the presence of inflammatory cells and mitoses suggest NF (2).

The best treatment is complete surgical excision with negative surgical margins (5). Awareness of nodular fasciitis and its benign nature is very important to avoid misdiagnosis and overtreatment for the patient. Since very few of these masses occur in children and the head and neck region, clinicians should consider this when evaluating pediatric soft tissue masses.

\section{Main Points}

1) Nodular fasciitis is characterized by rare, benign, proliferative masses localized in the subcutaneous tissue.

2) It presents as painless, rapid-growing solitary tumours.

3) It is commonly misdiagnosed as sarcoma because of rapid growth and increased mitotic activity.

4) The best treatment is complete surgical excision.

Informed Consent: Written informed consent was obtained.

Peer-Review: Externally peer-reviewed.

Author Contributions: Conception/Design of Study- M.C.K., I.Y., E.Y., A.B., M.Ü.; Data Acquisition- M.C.K., I.Y., E.Y., A.B., M.Ü.; Data Analysis/ Interpretation- M.C.K., İ.Y., E.Y., A.B., M.Ü.; Drafting Manuscript- M.C.K., I.Y., E.Y., A.B., M.Ü.; Critical Revision of Manuscript- M.C.K., I.Y., E.Y., A.B., M.Ü.; Final Approval and Accountability- M.C.K., I.Y., E.Y., A.B., M.Ü.

Conflict of Interest: Authors declared no conflict of interest.

Financial Disclosure: Authors declared no financial support.

\section{REFERENCES}

1. Be K. Subcutaneous pseudosarcomatous fibromatosis (fasciitis). Am J Clin Pathol 1955;25(3):241-52.

2. Allison DB, Wakely Jr PE, Siddiqui MT, Ali SZ. Nodular fasciitis: A frequent diagnostic pitfall on fine-needle aspiration. Cancer Cytopathol 2017;125(1):20-9.

3. DiNardo LJ, Wetmore RF, Potsic WP. Nodular fasciitis of the head and neck in children: a deceptive lesion. Arch Otolaryngol Neck Surg 1991;117(9):1001-2.

4. Erickson-Johnson MR, Chou MM, Evers BR, Roth CW, Seys AR, Jin L, et al. Nodular fasciitis: a novel model of transient neoplasia induced by MYH9-USP6 gene fusion. Lab Investig 2011;91(10):1427-33.

5. Oh BH, Kim J, Zheng Z, Roh MR, Chung KY. Treatment of nodular fasciitis occurring on the face. Ann Dermatol 2015;27(6):694-701. 